\title{
Early manifestation of alteration in cardiac function in dystrophin deficient mdx mouse using 3D CMR tagging Wei Li1,4, Wei Liu ${ }^{5}$, Jia Zhong ${ }^{1,4}$ and Xin Yu*1,2,3,4
}

Address: ${ }^{1}$ Department of Biomedical Engineering, Case Western Reserve University, Cleveland, Ohio, USA, ${ }^{2}$ Department of Radiology, Case Western Reserve University, Cleveland, Ohio, USA, ${ }^{3}$ Department of Physiology and Biophysics, Case Western Reserve University, Cleveland, Ohio, USA, ${ }^{4}$ Case Center for Imaging Research, Case Western Reserve University, Cleveland, Ohio, USA and ${ }^{5}$ Department of Biomedical Engineering, Washington University, St Louis, Missouri, USA

Email: Wei Li - wei.li2@case.edu; Wei Liu -wei.liu_1@philips.com; Jia Zhong - jia.zhong@case.edu; Xin Yu* - xin.yu@case.edu

* Corresponding author

Published: 22 October 2009

Journal of Cardiovascular Magnetic Resonance 2009, II:40 doi: I0.1 I86/1532-429X-II-40

This article is available from: http://www.jcmr-online.com/content/II/I/40

(C) 2009 Li et al; licensee BioMed Central Ltd.

This is an Open Access article distributed under the terms of the Creative Commons Attribution License (http://creativecommons.org/licenses/by/2.0), which permits unrestricted use, distribution, and reproduction in any medium, provided the original work is properly cited.
Received: 14 April 2009

Accepted: 22 October 2009

\begin{abstract}
Background: Duchenne muscular dystrophy (DMD) is caused by the absence of the cytoskeletal protein, dystrophin. In DMD patients, dilated cardiomyopathy leading to heart failure may occur during adolescence. However, early cardiac dysfunction is frequently undetected due to physical inactivity and generalized debilitation. The objective of this study is to determine the time course of cardiac functional alterations in $\mathrm{mdx}$ mouse, a mouse model of DMD, by evaluating regional ventricular function with CMR tagging.
\end{abstract}

Methods: In vivo myocardial function was evaluated by 3D CMR tagging in $\mathrm{mdx}$ mice at early (2 months), middle (7 months) and late (I0 months) stages of disease development. Global cardiac function, regional myocardial wall strains, and ventricular torsion were quantified. Myocardial lesions were assessed with Masson's trichrome staining.

Results: Global contractile indexes were similar between $\mathrm{mdx}$ and C57BL/6 mice in each age group. Histology analysis showed that young $\mathrm{mdx}$ mice were free of myocardial lesions. Interstitial fibrosis was present in 7 month $\mathrm{mdx}$ mice, with further development into patches or transmural lesions at 10 months of age. As a result, 10 month $\mathrm{mdx}$ mice showed significantly reduced regional strain and torsion. However, young $\mathrm{mdx}$ mice showed an unexpected increase in regional strain and torsion, while 7 month mdx mice displayed similar regional ventricular function as the controls.

Conclusion: Despite normal global ventricular function, CMR tagging detected a biphasic change in myocardial wall strain and torsion, with an initial increase at young age followed by progressive decrease at older ages. These results suggest that CMR tagging can provide more sensitive measures of functional alterations than global functional indexes in dystrophin-related cardiomyopathies.

\section{Background}

Duchenne muscular dystrophy (DMD) is an X-linked severe progressive muscle wasting disease, which affects approximately 1 in 3500 male birth [1,2]. DMD is caused by the deficiency of a cytoskeletal protein, dystrophin, which is a component of the transmembrane dystrophinglycoprotein complex (DGC). DGC plays an important role in maintaining the structural integrity of the cells by 
linking intracellular actin filaments to basal lamina [3,4]. The disruption of DGC structure due to dystrophin deficiency leads to dilated cardiomyopathy (DCM) that may occur during adolescence [5]. At an early stage, DMD patients usually do not present clinical cardiac symptoms because of their physical inactivity and generalized debilitation. However, a recent cardiovascular magnetic resonance (CMR) tagging study suggests that abnormal myocardial strain may develop long before the manifestation of global functional deterioration [6].

The $m d x$ mouse carries a nonsense mutation in its dystrophin gene that eliminates the expression of the dystrophin protein. It thus has been a popular animal model for studying the pathophysiology of DMD $[7,8]$. The $m d x$ mice exhibit many of the same histological features seen in $\mathrm{DMD}$, including the degeneration and necrosis of myofibers with inflammatory infiltrates, followed by subsequent regeneration. However, disease progression in $m d x$ mice is milder as compared to DMD patients. Only in relatively older $m d x$ mice are the progressive degenerative changes observed [9]. Similarly, $m d x$ mice manifest histological evidence of a cardiomyopathy, but no overt cardiac dysfunction has been found in the young $\mathrm{mdx}$ mice. Reduced global cardiac function [10] and dilated right ventricles [11] were only documented in relatively older mdx mice. In the current study, we sought to delineate alterations in cardiac function and structure during the course of DCM progression in mdx mice. Longitudinal evaluation of 3D regional myocardial wall motion, a potentially more sensitive measure of ventricular contractile behaviour, was performed with CMR tagging.

\section{Methods CMR}

In vivo CMR was performed at the Biological MR Laboratory of Washington University Medical Center. Mdx mice and their age-matched C57BL/ 6 wildtype controls of 2, 7 and 10 months of age underwent CMR on a 4.7 T Varian INOVA system (Varian Associates, Palo Alto, CA) equipped with a gradient insert $(60 \mathrm{G} / \mathrm{cm}, 10 \mathrm{~cm}$ inner diameter) as described previously [12]. A 2.5-cm surface coil was used for the imaging of mice. Briefly, mice were anesthetized with $0.7 \sim 1 \%$ isoflurane by a nose cone and placed into the coil in prone position. Electrodes were attached to front paws and the right leg for ECG gating and monitoring of vital signs. The animals were kept warm by blowing hot air into the magnet using a blow dryer. The heat flow and anaesthesia level were manually adjusted to keep the heart rate similar to that under conscious conditions. Animals were sacrificed at the end of imaging protocol for histological analysis. A total of 42 mice were scanned. The animal protocol was approved by the Animal Studies Committee of the Washington University Medical Center.
Tagged images of 5 short-axis (SA) slices were acquired from base to apex. The tagging sequence used a SPAMM1331 sequence applied immediately after the ECG trigger, followed by gradient-echo cine sequence. Repetition time (TR) was adjusted according to the R-R interval of the heart such that a total of 15 frames were acquired during one cardiac cycle. Other imaging parameters were: echotime (TE), 3 msec; data matrix, $256 \times 128$; field of view (FOV), $4 \mathrm{~cm} \times 4 \mathrm{~cm}$; slice thickness, $1 \mathrm{~mm}$. Tag resolution was $0.6 \mathrm{~mm}$ with tagline thickness ranging from 0.2 to 0.3 $\mathrm{mm}$. The acquired $k$-space data were zero-filled to yield a matrix size of $512 \times 512$, corresponding to an in-plane resolution of $78 \mu \mathrm{m} \times 78 \mu \mathrm{m}$. Two sets of tagged SA images were acquired with tags in perpendicular directions for each SA plane, yielding a grid tagging pattern when the two data sets were combined. Tagged long-axis (LA) images were acquired from four radially distributed LA views spaced every $45^{\circ}$, with the tags perpendicular to the $\mathrm{LV}$ long-axis. Imaging parameters were the same as those used for SA images.

Regular cine images that provided better contrast between the myocardium and the blood were acquired for morphological analysis. These images were acquired with the same imaging parameters as the tagged images with a 128 $\times 128$ data matrix. Both the cine and the tagged images were zero-filled into a $512 \times 512$ data matrix so that myocardial contours traced from cine images were directly used in the analysis of tagged images with minimal adjustment. Total image acquisition time, including animal setup, was $\sim 2$ hours.

\section{Image Analysis}

CMR data were analyzed with an in-house developed MATLAB-based software (CVMRI) described previously [12-15]. Epicardial and endocardial borders for SA images were traced interactively for the calculation of stroke volume, ejection fraction, and wall thickness. The left ventricular wall was further divided into four segments: septum, posterior, lateral, and anterior. Taglines for both SA and LA images were tracked semi-automatically with a HARP-based approach (Fig. 1A, B). Reconstruction of the $3 \mathrm{D}$ tag points was performed by first registering the SA and LA images in a 3D Cartesian coordinate system. Displacement of the intersecting tag points were tracked using an iterative point-tracking technique described previously [12]. The left ventricle was then divided into tetrahedrons using four non-coplanar adjacent tag points as the vertices. The 3D Lagrangian strain tensor was calculated from the deformation of these tetrahedrons, which was further diagonalized to yield principal strains $E_{1}, E_{2}$, and $\mathrm{E}_{3}$, with $\mathrm{E}_{1}$ and $\mathrm{E}_{3}$ being the maximal and minimal strains, respectively. The strain tensor was also transformed into a local myocardial coordinate system defined by the radial, circumferential, and longitudinal directions, 

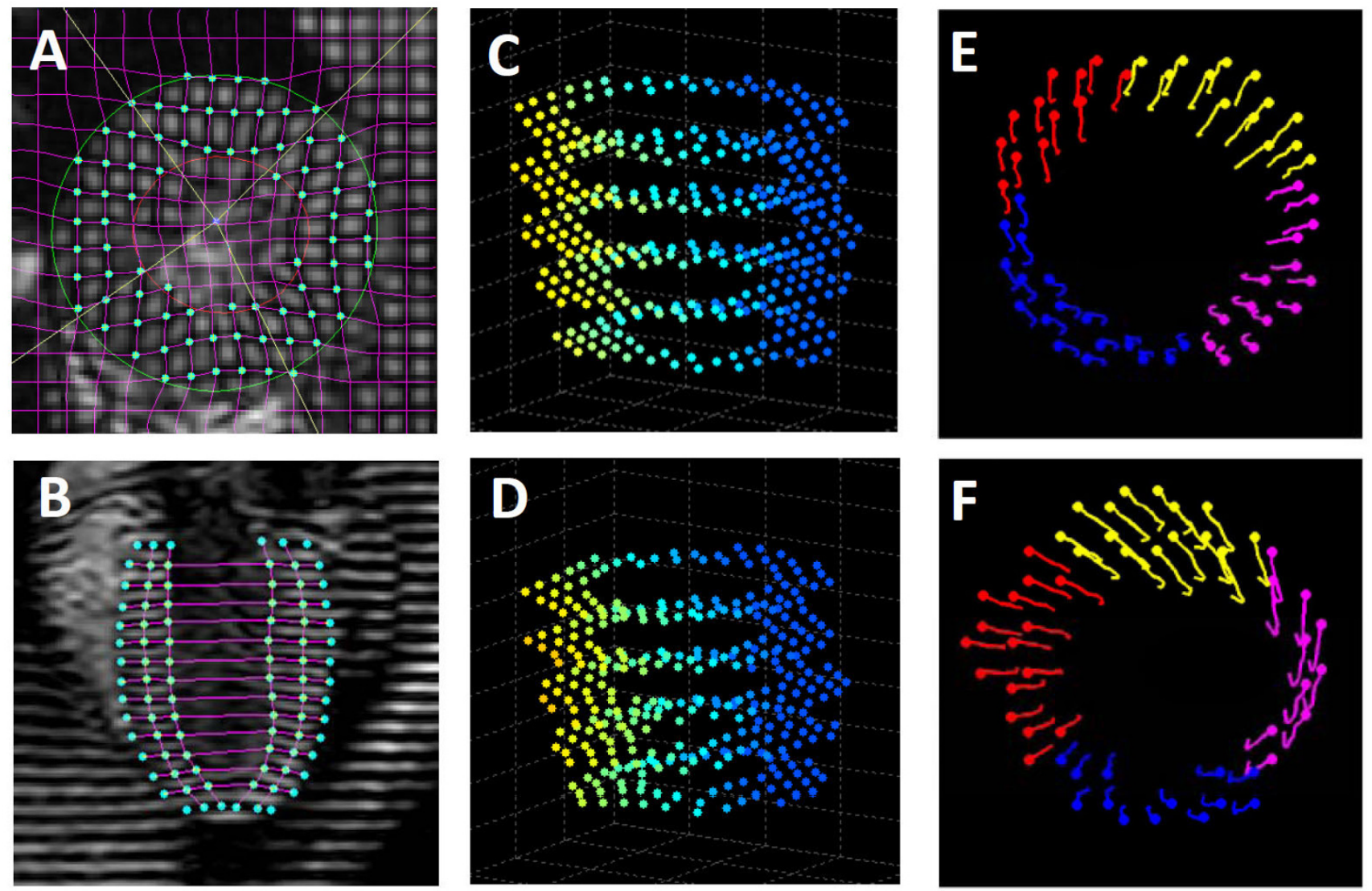

Figure I

3D MRI tagging image analysis. A\&B. Tracking of taglines in tagged short- and long-axis images, respectively; C\&D. Reconstructed 3D tag points at end-diastole and end-systole, respectively; E\&F. Trajectories of regional myocardial wall motion from end-diastole (dots) to end-systole (tails) at base and apex, respectively.

yielding three normal strains in the circumferential $\left(\mathrm{E}_{\mathrm{cc}}\right)$, radial $\left(\mathrm{E}_{\mathrm{rr}}\right)$ and longitudinal $\left(\mathrm{E}_{\mathrm{ll}}\right)$ directions, respectively. Myocardial twist was computed as the rotation angle around the center of LV cavity (Fig. 1E \&1F). Net twist angle was defined as the difference between the ventricular twist at apical and basal slices. Torsion was calculated as the net twist angle normalized by the slice separation. Typical processing time for a complete dataset was $\sim 3$ hours.

\section{Histology}

Upon the completion of CMR, the mouse was sacrificed with an overdose of $\mathrm{KCl}$ injection. The heart was excised and fixed in neutral-buffered $10 \%$ formalin for histological analysis. The fixed heart was sliced in 1-mm increment from base to apex along the LV long-axis. Each slice was embedded in paraffin and sectioned at $4 \mu \mathrm{m}$. Tissue sections were stained with Masson's trichrome for the identification of fibrotic lesions. The extent of myocardial fibrosis was quantified from histological images using a thresholding method developed in MATLAB (Fig. 2). Pix- els representing fibrotic tissue were extracted from the difference image of the red and blue channels of the original image, which provided the best contrast between normal and fibrotic tissue (Fig. 2C). Total myocardial tissue (normal and fibrotic) was extracted from the grayscale image (Fig. 2B). The percentage of tissue fibrosis was calculated as the ratio of the pixel numbers representing fibrotic and total myocardial tissue, respectively.

\section{Statistics}

Data are presented as mean \pm standard deviation. Unpaired Student t-test was used for comparisons between mdx mice and their age-matched controls. Mean values of myocardial strain in septal, anterior, lateral, and posterior segments, or at basal, midventricular, and apical levels, were compared separately by one-way analysis of variance (ANOVA). If there were statistical differences, multiple pairwise comparisons were performed using Tukey's test with a confidence interval of 95\%. P values less than 0.05 were considered statistically significant. 
A

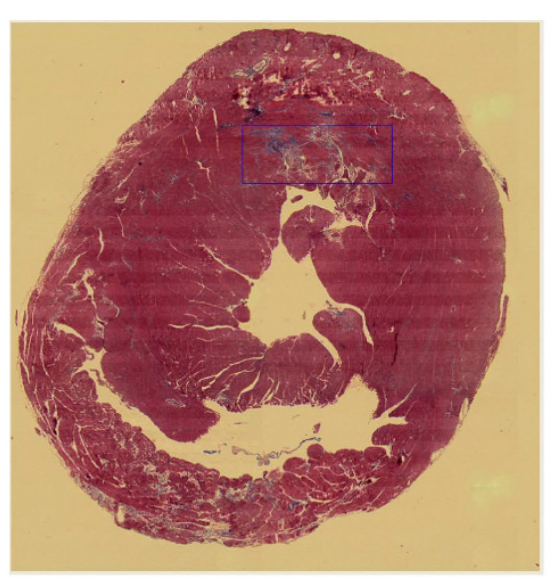

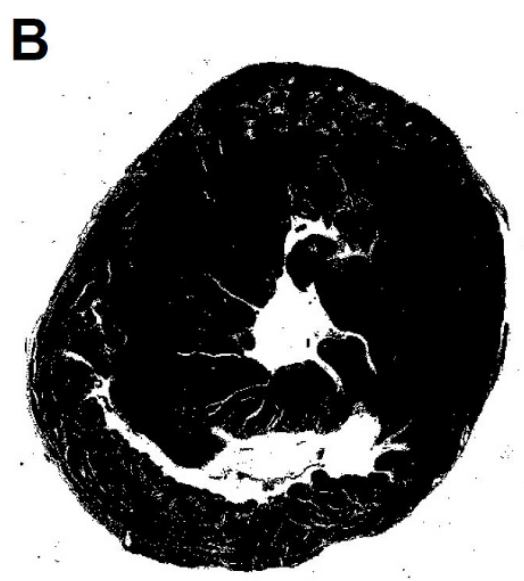

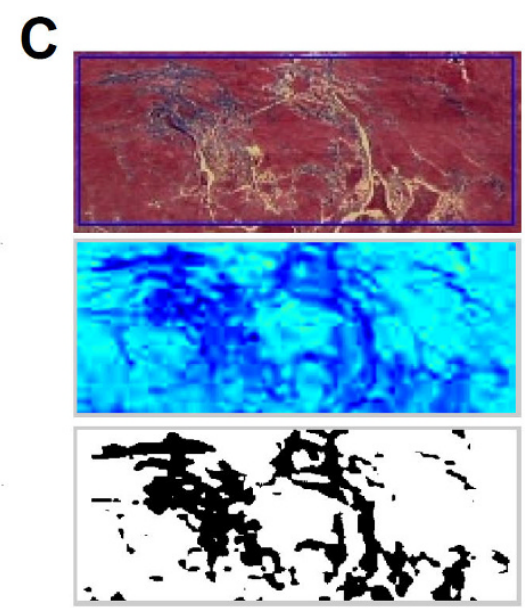

\section{Figure 2}

Quantification of myocardial fibrosis. A. Original histological image; B. Binary image of total tissue; C. Quantification of fibrotic tissue within a selected region of interest. Upper panel: original image; mid panel: pseudo-colored image of the difference between the red and blue channels; lower panel: binary image of fibrotic tissue.

\section{Results}

Animal Characteristics and Global Contractile Indexes The animal characteristic data and global ventricular function measured from CMR are summarized in Table 1. Heart rate was similar to that reported in the literature at normal body temperature [16-18]. Heart rate, LV enddiastolic volume (EDV), LV end-systolic volume (ESV), stroke volume, ejection fraction and cardiac output were all similar between mdx mice and their age-matched controls in all three age groups. 10-month mdx mice showed an increase in both heart weight and body weight, but similar heart weight to body weight ratio. In addition, there was also a slight increase in wall thickness in 10- month mdx mice. However, the wall thickness to LV diameter ratio was similar.

\section{2-month mdx mice}

Peak principal strain, normal strain, and ventricular torsion are shown in Figs. 3, 4 and 5 respectively. 2-month mdx mice had significantly increased regional wall strain and torsion. Compared with the control mice, mdx mice had a $12-44 \%$ increase in $\mathrm{E}_{2}$ ( $\mathrm{P}<0.05$ in two apical slices), a $9-16 \%$ increase in $\mathrm{E}_{3}(\mathrm{P}<0.05$ in three midventricular slices), and a $27-50 \%$ increase in $\mathrm{E}_{\mathrm{cc}}(\mathrm{P}<0.05$ for all 5 slices). Increased $\mathrm{E}_{1}$ was also observed in the slice between the mid-ventricle and the base in mdx mice $(\mathrm{P}<0.05)$. Ventricular torsion was also significantly increased in $\mathrm{mdx}$

Table I: Animal Characteristics and Morphological Data.

\begin{tabular}{|c|c|c|c|c|c|c|}
\hline & \multicolumn{2}{|c|}{2 month } & \multicolumn{2}{|c|}{7 month } & \multicolumn{2}{|c|}{10 month } \\
\hline & control $(n=8)$ & $\operatorname{mdx}(n=8)$ & control $(n=5)$ & $m d x(n=6)$ & control $(n=7)$ & $\operatorname{mdx}(n=8)$ \\
\hline Age, weeks & $8.5 \pm 1.3$ & $9.2 \pm 1.5$ & $30.6 \pm 1.8$ & $33.7 \pm 0.7$ & $45.2 \pm 2.8$ & $43.5 \pm 1.2$ \\
\hline Body weight, g & $26.9 \pm 1.8$ & $23.5 \pm 4.1$ & $32.2 \pm 5.3$ & $34.2 \pm 1.0$ & $30.0 \pm 2.9$ & $34.5 \pm 2.0 \ddagger$ \\
\hline Heart weight, mg & $|4| \pm 20$ & $127 \pm 13$ & $137 \pm 6$ & $158 \pm 29$ & $148 \pm 12$ & $170 \pm 12 \ddagger$ \\
\hline Heart weight/body weight, \% & $0.52 \pm 0.05$ & $0.55 \pm 0.13$ & $0.43 \pm 0.06$ & $0.46 \pm 0.09$ & $0.49 \pm 0.03$ & $0.49 \pm 0.03$ \\
\hline Heart rate, BPM & $466 \pm 42$ & $44 I \pm 40$ & $478 \pm 38$ & $481 \pm 54$ & $483 \pm 29$ & $500 \pm 22$ \\
\hline LV diameter, mm & $5.37 \pm 0.18$ & $5.07 \pm 0.35$ & $5.43 \pm 0.19$ & $5.7 I+0.12$ & $5.73 \pm 0.25$ & $5.75 \pm 0.08$ \\
\hline Wall thickness, mm & $0.97 \pm 0.16$ & $0.81 \pm 0.05$ & $1.02 \pm 0.08$ & $1.04 \pm 0.09$ & $0.94 \pm 0.04$ & $1.04 \pm 0.09 *$ \\
\hline Wall thickness/diameter & $0.18 \pm 0.03$ & $0.16 \pm 0.01$ & $0.20 \pm 0.03$ & $0.18 \pm 0.02$ & $0.17 \pm 0.01$ & $0.19 \pm 0.02$ \\
\hline LVEDV, $\mu \mathrm{l}$ & $42.8 \pm 6.7$ & $40.6 \pm 7.4$ & $42.1 \pm 7.4$ & $48.2 \pm 6.7$ & $51.0 \pm 7.2$ & $47.8 \pm 4.2$ \\
\hline LVESV, $\mu \mathrm{l}$ & $15.3 \pm 5.0$ & $13.7 \pm 3.5$ & $18.6 \pm 8.4$ & $20.9 \pm 5.3$ & $24.0 \pm 6.7$ & $24.3 \pm 2.9$ \\
\hline Stroke volume, $\mu \mathrm{l}$ & $27.5 \pm 3.1$ & $26.9 \pm 4.3$ & $25.9 \pm 0.5$ & $27.3 \pm 2.3$ & $27.0 \pm 3.2$ & $23.5 \pm 3.1$ \\
\hline Ejection fraction, \% & $65 \pm 7$ & $66 \pm 4$ & $63 \pm 10$ & $57 \pm 5$ & $54 \pm 8$ & $49 \pm 5$ \\
\hline Cardiac output, $\mathrm{ml} / \mathrm{min}$ & $12.9 \pm 1.5$ & $11.8 \pm 1.6$ & $12.7 \pm 0.9$ & $13.4 \pm 1.3$ & $13.0 \pm 2.2$ & $11.7 \pm 1.4$ \\
\hline
\end{tabular}

$* \mathrm{P}<0.05, \ddagger \mathrm{P}<0.0$ I between $\mathrm{mdx}$ mice and age matched controls. 
mice. The maximal torsion increased from $2.55 \pm 0.41^{\circ} \%$ $\mathrm{mm}$ in control mice to $3.40 \pm 0.83^{\circ} / \mathrm{mm}$ in $\mathrm{mdx}$ mice (P $<0.05)$.

\section{7-Month Mdx Mice}

7-month mdx mice had similar regional strain and time course of ventricular torsion as the controls. The maximal torsion of $\mathrm{mdx}$ mice was also similar to the controls $(2.55$ $\pm 0.25^{\circ} / \mathrm{mm}$ v.s. $\left.3.06 \pm 0.97^{\circ} / \mathrm{mm}, \mathrm{P}=0.3\right)$.

\section{I0-Month Mdx Mice}

10-month mdx mice had significantly decreased regional myocardial wall strains $\left(\mathrm{E}_{1}, \mathrm{E}_{2}, \mathrm{E}_{3}, \mathrm{E}_{\mathrm{cc}} \mathrm{E}_{\mathrm{rr}}\right.$ and $\left.\mathrm{E}_{\mathrm{ll}}\right)$ by $11 \sim 38 \%$ throughout the whole left ventricle, with the exception of $E_{1}$ at base, $E_{l l}$ at midventricle, and $E_{r r}$ at both apex and base. The time course of ventricular torsion was significantly altered in mdx mice (Fig. 5C). The maximal torsion decreased from $2.65 \pm 0.86^{\circ} / \mathrm{mm}$ in the control mice to $1.66 \pm 0.40^{\circ} / \mathrm{mm}$ in $\mathrm{mdx}$ mice $(\mathrm{P}<0.05)$.

\section{Histological Analysis and Principal Strains in Segmented Regions}

Representative histological images are shown in Fig. 6. 2month mdx hearts did not show obvious cardiac lesions $(0.6 \pm 0.5 \%) .7$-month $\mathrm{mdx}$ hearts had mild interstitial fibrotic lesions $(3.8 \pm 1.1 \%)$. 10-month mdx hearts had a wide range of tissue fibrosis from interstitial to transmural fibrotic patches. The fibrotic tissue averaged $7.6 \pm 3.9 \%$ in 10 -month mdx mice. The fibrotic tissue displayed a random and scattered pattern. Myocardial fibrosis was observed in the septum, anterior, posterior and lateral segments of the left ventricle, as well as in the right ventricle.

Principal strains at apex, mid-ventricle, and base are shown in Fig. 7. For 2-month $\mathrm{mdx}$ mice, anterior $\mathrm{E}_{1}$ strain was decreased and lateral $E_{2}$ strain was increased in the apex, posterior $\mathrm{E}_{2}$ and $\mathrm{E} 3$ strains were increased in midventricle, as well as posterior $\mathrm{E}_{2}$ strain in the base $(\mathrm{P}<$ $0.05)$. For 7 -month $\mathrm{mdx}$ mice, septum $\mathrm{E}_{1}$ strain was

A A
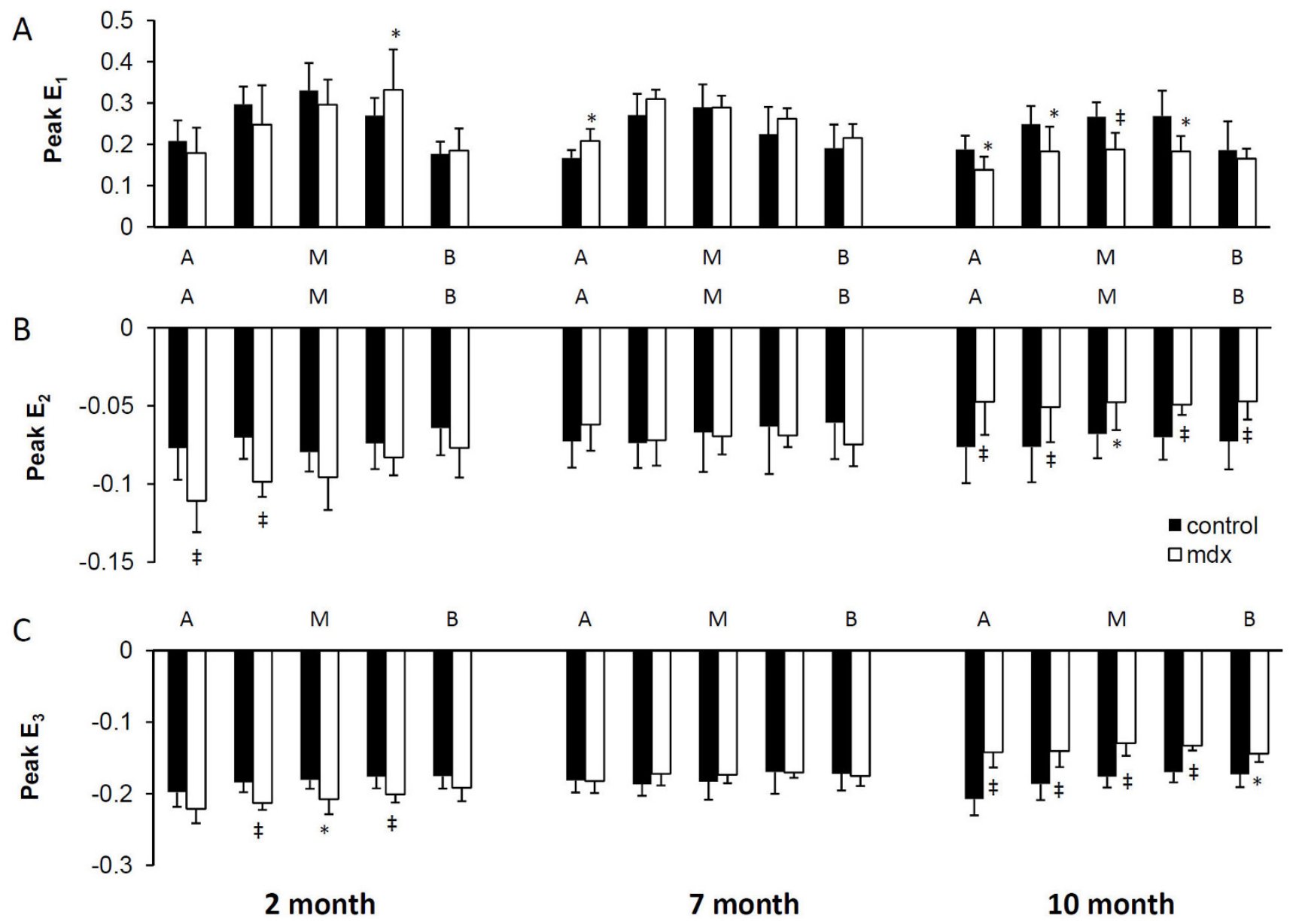

Figure 3

Peak principal strains. Peak $E_{1}(A), E_{2}(B)$, and $E_{3}(C)$ strains in 5 short-axis levels from apex $(A)$, to mid-ventricle $(M)$, and to base $(\mathrm{B})$. $* \mathrm{P}<0.05, \neq \mathrm{P}<0.0 \mathrm{I}$ between $\mathrm{mdx}$ mice and age-matched controls, respectively. 


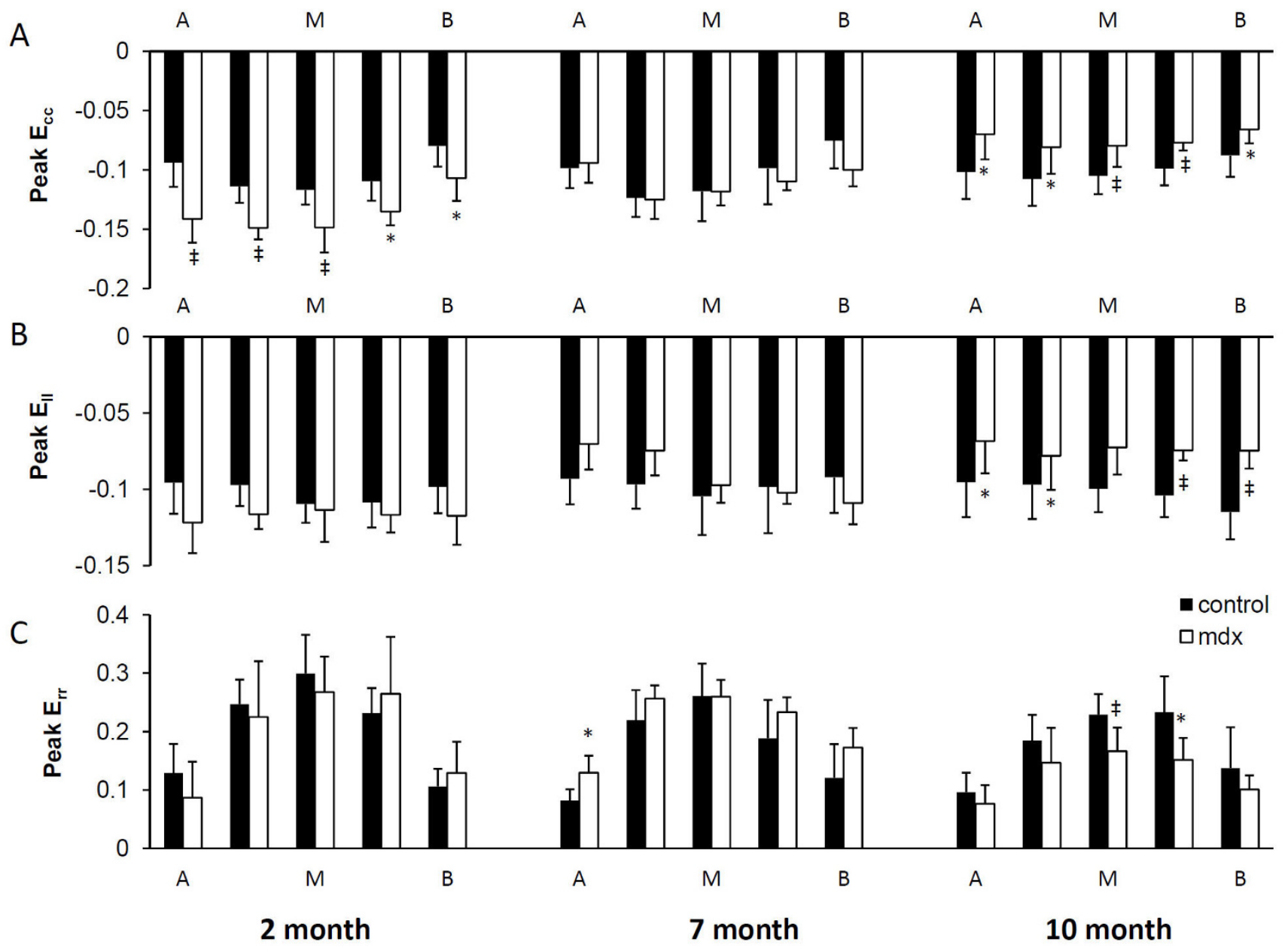

Figure 4

Peak normal strains. Peak circumferential (A), longitudinal (B), and radial (C) strains in 5 short-axis levels from apex (A), to mid-ventricle $(\mathrm{M})$, and to base $(B)$. ${ }^{*}<0.05, \pm P<0.01$ between $\mathrm{mdx}$ mice and age-matched controls, respectively.

increased at the apex, while lateral $\mathrm{E}_{3}$ strain was decreased in mid-ventricle. At 10 months of age, all the segments showed either a decrease or a trend towards decrease in three principal strains in mdx mice. Consistent with the scattered fibrosis, all four segments of the left ventricle had a decrease in wall strain in 10-month mdx mice.

\section{Discussion}

In this study in mdx mice, we assessed the alterations in regional left ventricular function at different stages of disease development towards DCM. Despite similar global functional indexes, both young and adult mdx mice manifested significantly altered patterns of regional ventricular wall motion. Consistent with the development of fibrotic lesions, myocardial wall strain and torsion were significantly decreased in 10-month mdx mice. However, the lesion-free young mdx mice had a surprising increase in regional wall strain and torsion, while 7 -month mdx mice had similar contractile patterns as the controls.

Cardiac function has been investigated in mdx mice using both CMR and echocardiography. Young mdx mice exhibited normal morphology and global cardiac function $[10,19]$. At 8 months of age, mdx mice started to manifest right ventricular dilatation and decreased peak LV filling rate [11]. However, ejection fraction and cardiac output in mdx mice remained normal until 42 weeks of age $[10,11]$. While the normal global function in young and adult $\mathrm{mdx}$ mice is consistent with these previous findings, our study is the first to observe an early change in regional myocardial wall strain and torsion in mdx mice. Our results suggest that changes in regional wall motion can occur at a very early age in mdx mice before any macroscopic and global functional changes have occurred. 
A

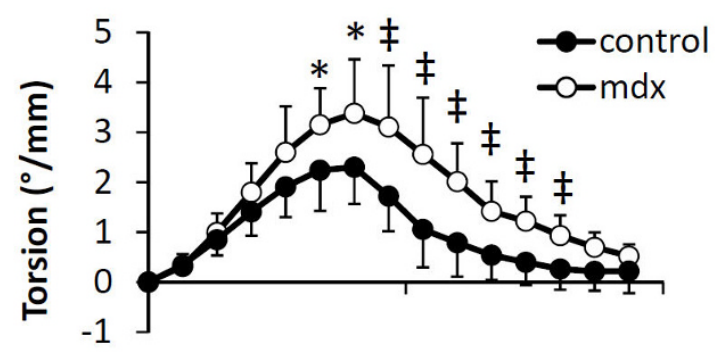

B

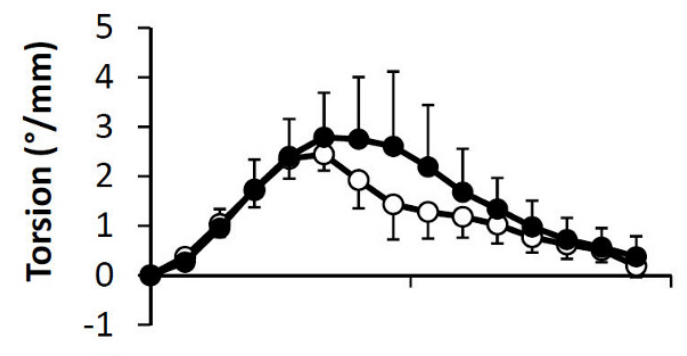

C

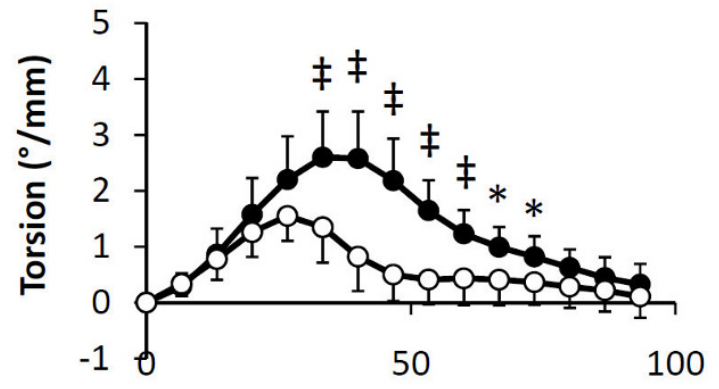

$\%$ cardiac cycle

Figure 5

Time course of ventricular torsion. A. Increased ventricular torsion in 2-month $\mathrm{mdx}$ mice; B. Similar ventricular torsion between $\mathrm{mdx}$ and control mice at 7 month; $\mathrm{C}$. Decreased ventricular torsion in 10 -month $\mathrm{mdx}$ mice. $* \mathrm{P}<$ 0.05 , $¥ \mathrm{P}<0.0$ l between $\mathrm{mdx}$ mice and age-matched controls, respectively.

We observed a biphasic change in myocardial wall strain and torsion in mdx mice, with an initial increase at young age followed by decrease at older ages. Since dystrophin disruption renders $\mathrm{mdx}$ mice more vulnerable to mechanical stress and workload induced damage [20], the early increase in myocardial contraction may lead to increased ventricular wall stress in $\mathrm{mdx}$ mice, thus exacerbating stress-induced damage and triggering the initial remodeling processes that eventually leads to lesion development and cardiac dysfunction. The accumulation of tissue fibrosis leads to progressive decline in regional ventricular function in older mdx mice. As a result, 7-month $\mathrm{mdx}$ mice had similar regional ventricular function compared with the controls, while 10-month mdx mice had significantly decreased wall strain and torsion.
Cellular changes in mdx mice have also been investigated. In particular, the impact of dystrophin deficiency on $\mathrm{Ca}^{2+}$ homeostasis has been investigated in both young (2-3 months) and adult (9-12 months) mdx mice by Williams et al [21]. They observed significantly increased $\mathrm{Ca}^{2+}$ transient in both young and adult mdx mice, which was associated with increased expression of ryanodine receptor, the protein responsible for calcium release during systole. As $\mathrm{Ca}^{2+}$ is the central regulator of myocyte contractility, it is possible that our observed functional increase in young mdx mice is attributable to enhanced myocyte contraction due to increased $\mathrm{Ca}^{2+}$ transients. However, further investigation is needed to establish the relationship of calcium transients, myocardial contractile function, and disease progression in mdx mice.

The effects of increased $\mathrm{Ca}^{2+}$ transients on myocardial remodelling have been investigated in other animal models. Increased $\mathrm{Ca}^{2+}$ transients and myocyte hypertrophy were observed in mice with $\beta_{2}$-adrenoceptor overexpression [22]. Increase in $\mathrm{Ca}^{2+}$ transients via systemic $\beta$-agonist administration has also been shown to cause myocyte hypertrophy and apoptosis, tissue fibrosis, and heart failure [23]. All these studies indicate a possible mechanism leading to myocardial dysfunction via chronic elevation of $\mathrm{Ca}^{2+}$ transients. Interestingly, altered $\mathrm{Ca}^{2+}$ homeostasis was also observed in the skeletal muscle of both $\mathrm{mdx}$ mice and DMD patients [24-26]. These observations led to several clinical trials that evaluated the effects of $\mathrm{Ca}^{2+}$ antagonists on DMD patients [27], with the primary focus on the skeletal muscle. In general, no significant beneficial effects were observed on the skeletal muscle function in DMD patients, although reduced muscle degeneration was revealed in several animal studies [28-30]. Only one study reported a significant improvement in muscle strength in verapamil-treated DMD patients, but with a high incidence of cardiac side effects [31]. Since $\mathrm{Ca}^{2+} \mathrm{can}$ impact both inotropic and chronotropic state of the heart, the effects of $\mathrm{Ca}^{2+}$ antagonists on the heart need to be carefully examined.

Several studies have investigated LV function in DMD patients in search for early signs of myocardial dysfunction. Using CMR, Ashford et al reported normal LV volume and ejection fraction in young DMD patients. However, CMR tagging revealed significantly reduced circumferential strain in these patients [6]. Similar findings were reported using ultrasound-based methods. Mertens et al investigated a group of young DMD patients with preserved global function by echocardiography [32]. They observed significantly decreased peak radial and longitudinal systolic strain and strain rate, as well as peak systolic and early diastolic myocardial velocities in the anterolateral and inferolateral LV walls. Similarly, Mori et al reported decreased peak systolic radial strain and early 

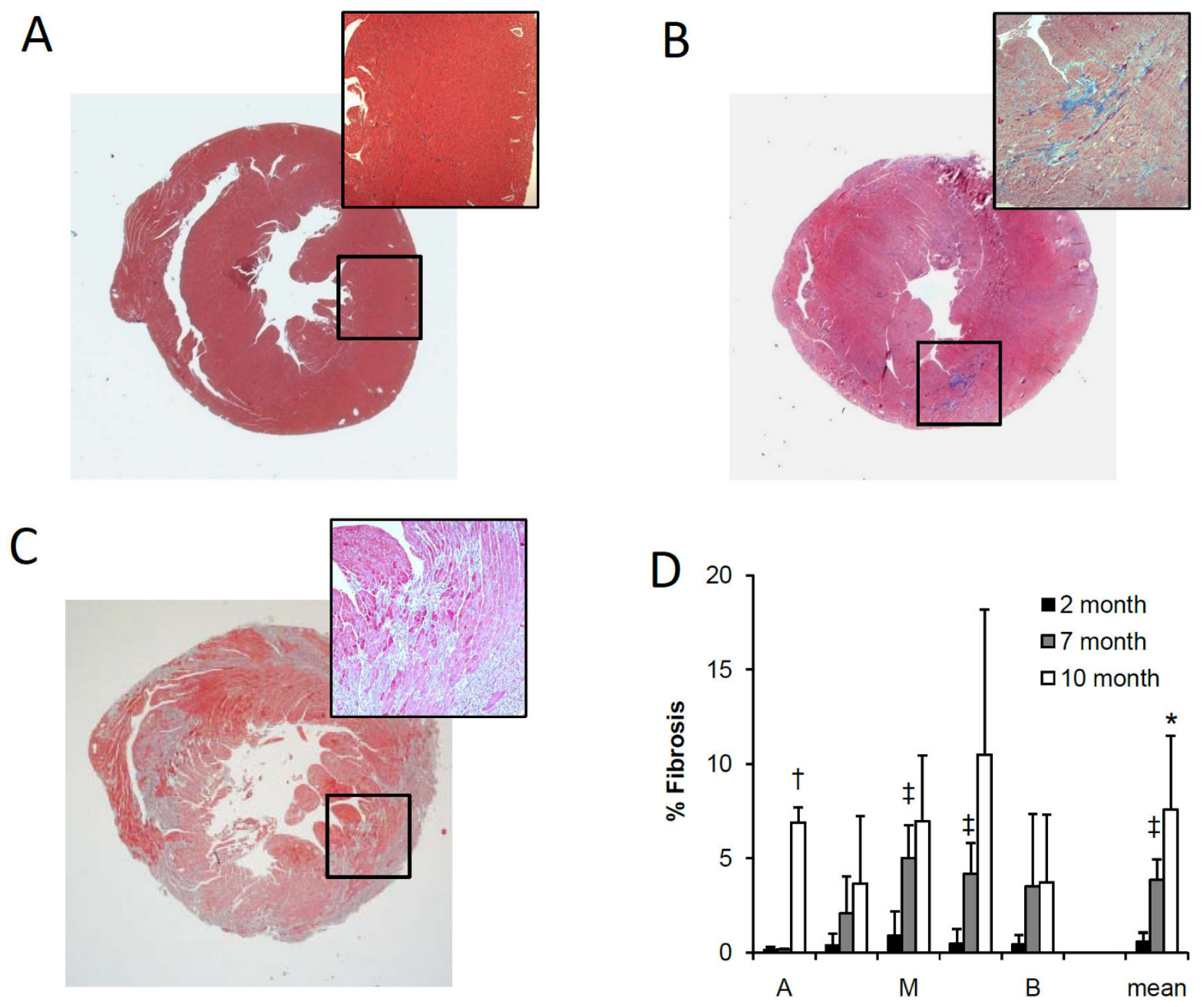

Figure 6

Histological assessment of $\mathbf{m d x}$ mouse hearts. Representative images of Masson's trichrome staining of $\mathrm{mdx}$ mouse hearts at $2(A), 7(B)$, and IO (C) months of age. D. Quantification of fibrotic tissue at five short-axis levels from apex (A) to midventricle $(M)$, and to base $(B)$. $\neq P<0.01$ between 2 - and 7 -month mdx mice. $* P<0.05$, $\nmid P<0$.0 I between 7 - and 10 -month mdx mice, respectively.

diastolic wall-thinning velocity in the posterior $\mathrm{LV}$ wall of young DMD patients with normal conventional echocardiographic findings $[33,34]$. These studies suggest that DMD patients manifest decreased regional myocardial wall motion at an early age, which differ from the biphasic change we observed in $\mathrm{mdx}$ mice. Compare to DMD patients, the expression of disease in $\mathrm{mdx}$ mice is relatively milder [35]. One possible explanation for this difference between mdx mice and DMD patients is that a dystrophin analogue, utrophin, compensates for the lack of dystrophin more effectively in mdx mice than in DMD patients. Utrophin is associated with the DGC, and is similar to dystrophin in primary sequence and predicted sec- ondary structure [36]. It can compensate for dystrophin when expressed at high levels. The severity of disease in mice lacking both dystrophin and utrophin is similar to DMD $[37,38]$. Further, a recent gene array study has confirmed the up-regulation of utrophin in mdx mice [39].

While the observed biphasic change in mdx mice suggests that tissue tagging is more sensitive for detecting functional alterations than global clinical indexes, functional assessment alone may not be sufficient in delineating disease progression when opposing effects exist. The seemingly "normal" regional wall motion in 7 -month $\mathrm{mdx}$ mice reflects the balance between increased myocyte con- 


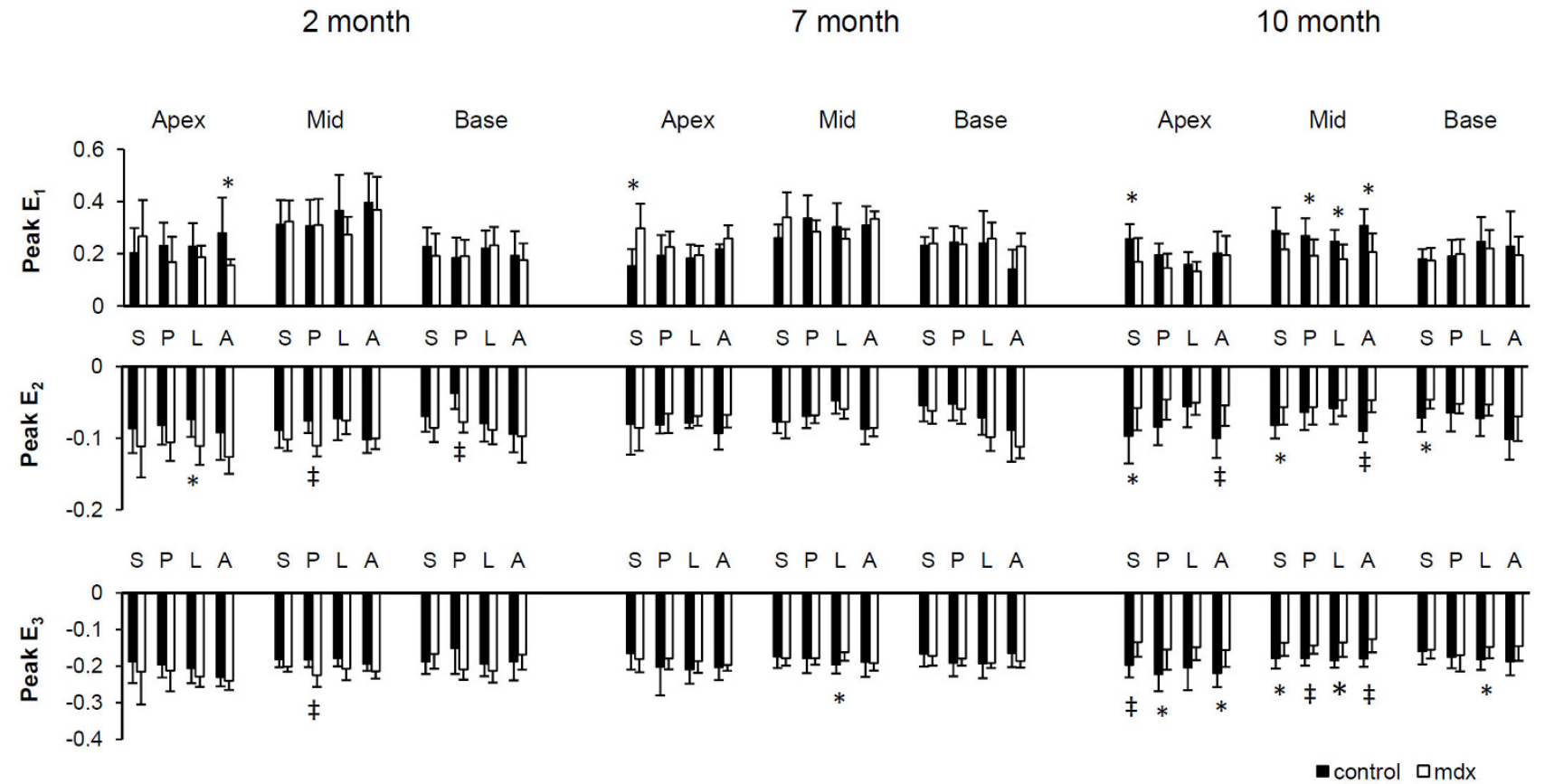

Figure 7

Principal strains in segmented regions. S, septum; $\mathrm{P}$, posterior; $\mathrm{L}$, lateral; $\mathrm{A}$, anterior. $* \mathrm{P}<0.05, \pm \mathrm{P}<0.0 \mathrm{I}$ between $\mathrm{mdx}$ mice and age-matched controls, prespectively.

tractility and the development of myocardial fibrosis. Because of these counteracting factors, it is important to evaluate other aspects of myocardial remodeling. In particular, late gadolinium enhanced CMR (LGE-CMR) has been shown to detect myocardial lesions in DMD patients [40-42]. The combination of LGE-CMR and tissue tagging will provide more comprehensive evaluation of disease progression in DMD patients.

Despite significant alterations in ventricular torsion and wall strain at both 2 - and 10-months of age, mdx mice showed no statistical difference in global ventricular function compared to the age-matched controls. The relationship between regional myocardial wall motion and global ventricular function is complicated because of the intricate 3D architecture of myocardial fibers. While CMR tagging measures the regional deformation of the myocardium, it remains to be elucidated to what extent altered myocardial deformation can be reflected in global changes in ventricular volumes. In general, current findings support the notion that regional alterations precede the manifestation of global functional changes. However, a more quantitative relationship between regional wall deformation and global ventricular function remains to be defined.

Nevertheless, older mdx mice showed a trend towards decreased ejection fraction (Table 1). Correlation analysis revealed a weak, negative correlation between tissue fibrosis and ejection fraction (Fig. 8). A recent study of DMD patients also investigated the correlation between ejection fraction and myocardial lesion delineated by LGE [42]. Although a reduction in ejection fraction was reported in DMD patients with significant lesion development (21.9 $\pm 11.1 \%$ ), no statistical significance was detected in ageadjusted correlation between ejection fraction and lesion size $(P=0.065)$. The mdx mice differ from DMD patients with delayed disease progression. With an average fibrosis of $7.6 \pm 3.9 \%$ in 10 -month $\mathrm{mdx}$ mice, the reduction in ejection fraction was insignificant. These data further suggest that analysis of regional myocardial wall motion can provide more sensitive measures of functional alterations in heart.

Previous LGE-CMR studies have identified the basal inferior and lateral walls as the initial sites of myocardial fibrosis $[41,42]$. A recent echocardiographic study also observed decreased radial strain in the posterior wall with relative sparing of ventricular septum [34]. However, such preferential changes were not observed by CMR tagging [6]. These discrepancies and the complicated relationship between myocardial fibre structure and regional function warrant further investigation. In the current study, we observed atypical patterns of lesion distribution and functional alterations in mdx mice. It remains to be investigated which pathogenic processes are responsible for the 


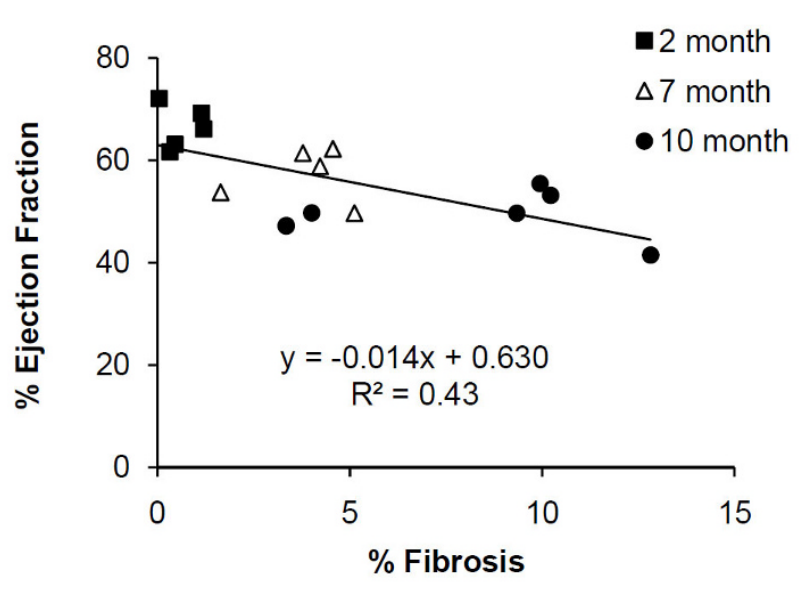

Figure 8

Correlation between ejection fraction and myocardial fibrosis.

observed difference between mdx mice and DMD patients.

One limitation of the current study is that different animals were characterized in different age groups because of the requirement for histological analysis, which may potentially increase the variability of strain measurements. Heart rate variation during long image acquisition can be a source of error for the $3 \mathrm{D}$ reconstruction of the taglines. In the current study, this error was minimized by maintaining a constant heart rate with $4.1 \pm 4.7 \%$ fluctuation. The TR was also adjusted according to the R-R interval such that each of the 15 frames was acquired at the same stage during a cardiac cycle. These measures can effectively minimize the errors due to heart rate fluctuation. The strain and torsion values in the control mice were similar to those reported in the literature $[16,43]$.

Taglines were tracked semi-automatically with HARPbased method and homogeneous strain analysis was used for strain quantification in the current study. While the filtering of harmonic peaks in the k-space reduces the resolution of reconstructed HARP images, the accuracy of tagline tracking is not affected by k-space sampling/undersampling. The accuracy of homogeneous strain analysis was investigated previously using a deformable silicone gel phantom [44]. It has been shown that the error of noise-free strain estimation was within $10 \%$ of the modelcalculated values, with no bias. With only one adjacent slice, strain quantification at apex showed a larger variation than at mid-ventricle as fewer tetrahedrons were generated. However, average strain values for each experimental group showed comparable standard deviations at apex, base, and mid-ventricle, suggesting that dif- ferences among animals were the dominant source of data variation.

\section{Conclusion}

Despite normal global LV function, mdx mice had a biphasic change in myocardial wall strain and torsion, with an initial increase at young age followed by progressive decrease at older ages. These results suggest that regional $\mathrm{LV}$ function by $\mathrm{CMR}$ tagging is sensitive to early signs of myocardial dysfunction than global functional indexes. Therefore, CMR tagging can be utilized to detect early cardiac involvement, as well as to potentially monitor and quantify the effects of novel therapeutic interventions in DMD patients.

\section{Competing interests}

The authors declare that they have no competing interests.

\section{Authors' contributions}

WLi participated in study design, image and histological analysis, and prepared the manuscript. WLiu participated in study design, image acquisition and analysis. JZ participated in developing the 3D tagging analysis method. XY conceived and supervised the study and participated in the writing of this manuscript. All authors read and approved the final manuscript.

\section{Acknowledgements}

The authors thank Dr. Joseph J.H. Ackerman, Director of the Biomedical MR Laboratory (BMRL) at Washington University, for advice and MR resources. The authors also thank Dr. Xiongwei Zhu from Department of Pathology at Case Western Reserve University for assistance with histological analysis. The authors acknowledge the support of the Washington University Small Animal Imaging Resource, funded in part through National Cancer Institute Small Animal Imaging Research Program Grant R24 CA83060.

This work was supported by NIH grants R0I HL-733I5, HL-86935 (X.Y.). Wei Liu and jia Zhong were supported by American Heart Association predoctoral fellowships $0215174 Z$ (to W.L.) and 0615308B (to J.Z.).

\section{References}

I. Koenig M, Beggs AH, Moyer M, Scherpf S, Heindrich K, Bettecken T, Meng G, Muller CR, Lindlof M, Kaariainen H, de la Chapelle A, Kiuru A, Savontaus M-L, Gilgenkrantz H, Recan D, Chelly J, Kaplan J-C, Covone AE, Archidiacono N, Romeo G, Liechti-Gallati S, Schneider V, Braga S, Moser H, Darras BT, Murphy P, Francke U, Chen JD, Morgan G, Denton M, Greenberg CR, Wrogemann K, Blonden LAJ, Van Ommen GJB, Kunkel LM: The molecular basis for Duchenne versus Becker muscular dystrophy: correlation of severity with type of deletion. Am J Hum Genet 1989, 45:498-506.

2. Biggar WD, Klamut HJ, Demacio PC, Stevens DJ, Ray PN: Duchenne muscular dystrophy: current knowledge, treatment, and future prospects. Clin Orthop Relat Res 2002, 40 I:88-106.

3. Blake DJ, Weir A, Newey SE, Davies KE: Function and genetics of dystrophin and dystrophin-related proteins in muscle. Physiol Rev 2002, 82:291-329.

4. Blake DJ, Martin-Rendon E: Intermediate filaments and the function of the dystrophin-protein complex. Trends Cardiovasc Med 2002, I 2:224-228.

5. Hoogerwaard EM, Wouw PA van der, Wilde AA, Bakker E, Ippel PF, Oosterwijk JC, Majoor-Krakauer DF, van Essen AJ, Leschot NJ, de 
Visser M: Cardiac involvement in carriers of Duchenne and Becker muscular dystrophy. Neuromuscul Disord 1999, 9:347-35I.

6. Ashford MW Jr, Liu W, Lin SJ, Abraszewski P, Caruthers SD, Connolly AM, Yu X, Wickline SA: Occult cardiac contractile dysfunction in dystrophin-deficient children revealed by cardiac magnetic resonance strain imaging. Circulation 2005, I I 2:2462-2467.

7. Sicinski P, Geng Y, Ryder-Cook AS, Barnard EA, Darlison MG, Barnard PJ: The molecular basis of muscular dystrophy in the mdx mouse: a point mutation. Science 1989, 244:1578-I580.

8. Ohlendieck K, Campbell KP: Dystrophin-associated proteins are greatly reduced in skeletal muscle from mdx mice. J Cell Biol I991, I I 5: 1685-1694.

9. Pastoret $C$, Sebille $A$ : $\mathbf{m d x}$ mice show progressive weakness and muscle deterioration with age. I Neurol Sci 1995, 1 29:97-105.

10. Quinlan JG, Hahn HS, Wong BL, Lorenz JN, Wenisch AS, Levin LS: Evolution of the mdx mouse cardiomyopathy: physiological and morphological findings. Neuromuscul Disord 2004, 14:49|-496.

II. Zhang W, ten Hove M, Schneider JE, Stuckey DJ, Sebag-Montefiore L, Bia BL, Radda GK, Davies KE, Neubauer S, Clarke K: Abnormal cardiac morphology, function and energy metabolism in the dystrophic mdx mouse: an MRI and MRS study. J Mol Cell Cardiol 2008, 45:754-760.

12. Zhong J, Liu W, Yu X: Characterization of three-dimensional myocardial deformation in the mouse heart: an MR tagging study. J Magn Reson Imaging 2008, 27: I 263-1270.

13. Liu W, Chen J, Ji S, Allen JS, Bayly PV, Wickline SA, Yu X: Harmonic phase MR tagging for direct quantification of Lagrangian strain in rat hearts after myocardial infarction. Magn Reson Med 2004, 52: $1282-1290$.

14. Liu W, Ashford MW, Chen J, Watkins MP, Williams TA, Wickline SA, Yu X: MR tagging demonstrates quantitative differences in regional ventricular wall motion in mice, rats, and men. $\mathrm{Am}$ J Physiol Heart Circ Physiol 2006, 29 I:H25 I5-H252I.

15. Zhong J, Liu W, Yu X: Transmural myocardial strain in mouse: quantification of high-resolution MR tagging using harmonic phase (HARP) analysis. Magn Reson Med 2009, 61:1368-1373.

16. Zhou R, Pickup S, Glickson JD, Scott CH, Ferrari VA: Assessment of global and regional myocardial function in the mouse using cine and tagged MRI. Magn Reson Med 2003, 49:760-764.

17. Epstein FH, Yang Z, Gilson WD, Berr SS, Kramer CM, French BA: MR tagging early after myocardial infarction in mice demonstrates contractile dysfunction in adjacent and remote regions. Magn Reson Med 2002, 48:399-403.

18. Vandsburger MH, French BA, Helm PA, Roy RJ, Kramer CM, Young AA, Epstein FH: Multi-parameter in vivo cardiac magnetic resonance imaging demonstrates normal perfusion reserve despite severely attenuated \{beta\}-adrenergic functional response in neuronal nitric oxide synthase knockout mice. Eur Heart J 2007, 28:2792-2798.

19. Wilding JR, Schneider JE, Sang AE, Davies KE, Neubauer S, Clarke K: Dystrophin- and MLP-deficient mouse hearts: marked differences in morphology and function, but similar accumulation of cytoskeletal proteins. FASEB J 2005, 19:79-8I.

20. Danialou G, Comtois AS, Dudley R, Karpati G, Vincent G, Des Rosiers C, Petrof BJ: Dystrophin-deficient cardiomyocytes are abnormally vulnerable to mechanical stress-induced contractile failure and injury. FASEB J 200 I, I 5: 1655-1657.

21. Williams IA, Allen DG: Intracellular calcium handling in ventricular myocytes from mdx mice. Am J Physiol Heart Circ Physiol 2007, 292: H846-H855.

22. Schwarz B, Percy E, Gao XM, Dart AM, Richardt G, Du XJ: Altered calcium transient and development of hypertrophy in \{beta\}2-adrenoceptor overexpressing mice with and without pressure overload. Eur J Heart Fail 2003, 5:131-136.

23. Osadchii OE: Cardiac hypertrophy induced by sustained betaadrenoreceptor activation: pathophysiological aspects. Heart Fail Rev 2007, I 2:66-86.

24. Fong PY, Turner PR, Denetclaw WF, Steinhardt RA: Increased activity of calcium leak channels in myotubes of Duchenne human and mdx mouse origin. Science 1990, 250:673-676.
25. Kumar A, Khandelwal N, Malya R, Reid MB, Boriek AM: Loss of dystrophin causes aberrant mechanotransduction in skeletal muscle fibers. FASEB J 2004, 1 8: I02-II3.

26. Bodensteiner JB, Engel AG: Intracellular calcium accumulation in Duchenne dystrophy and other myopathies: a study of $\mathbf{5 6 7 , 0 0 0}$ muscle fibers in 114 biopsies. Neurology 1978, 28:439-446

27. Phillips MF, Quinlivan R: Calcium antagonists for Duchenne muscular dystrophy. Cochrane Database Syst Rev 2008:CD00457I.

28. Matsumura CY, Pertille A, Albuquerque TC, Santo NH, Marques MJ: Diltiazem and verapamil protect dystrophin-deficient muscle fibers of MDX mice from degeneration: a potential role in calcium buffering and sarcolemmal stability. Muscle Nerve 2009, 39:167-176.

29. Mariol MC, Segalat L: Muscular degeneration in the absence of dystrophin is a calcium-dependent process. Curr Biol 200I, I I: $169 \mid-1694$.

30. Johnson PL, Bhattacharya SK: Regulation of membrane-mediated chronic muscle degeneration in dystrophic hamsters by calcium-channel blockers: diltiazem, nifedipine and verapamil. J Neurol Sci 1993, I I5:76-90.

31. Emery AE, Skinner R, Howden LC, Matthews MB: Verapamil in Duchenne muscular dystrophy. Lancet 1982, I:559.

32. Mertens L, Ganame J, Claus P, Goemans N, Thijs D, Eyskens B, Van Laere D, Bijnens B, D'hooge J, Sutherland GR, Buyse G: Early regional myocardial dysfunction in young patients with Duchenne muscular dystrophy. J Am Soc Echocardiogr 2008, 21:1049-1054.

33. Mori K, Edagawa T, Inoue M, Nii M, Nakagawa R, Takehara $Y$, Kuroda $Y$, Tatara K: Peak negative myocardial velocity gradient and wall-thickening velocity during early diastole are noninvasive parameters of left ventricular diastolic function in patients with Duchenne's progressive muscular dystrophy. J Am Soc Echocardiogr 2004, 17:322-329.

34. Mori K, Hayabuchi Y, Inoue M, Suzuki M, Sakata M, Nakagawa R, Kagami S, Tatara K, Hirayama Y, Abe Y: Myocardial strain imaging for early detection of cardiac involvement in patients with Duchenne's progressive muscular dystrophy. Echocardiography 2007, 24:598-608.

35. Banks GB, Chamberlain JS: The value of mammalian models for duchenne muscular dystrophy in developing therapeutic strategies. Curr Top Dev Biol 2008, 84:43I-453.

36. Blake DJ, Tinsley JM, Davies KE: Utrophin: a structural and functional comparison to dystrophin. Brain Pathol 1996, 6:37-47.

37. Grady RM, Teng H, Nichol MC, Cunningham JC, Wilkinson RS, Sanes JR: Skeletal and cardiac myopathies in mice lacking utrophin and dystrophin: a model for Duchenne muscular dystrophy. Cell 1997, 90:729-738.

38. Deconinck AE, Rafael JA, Skinner JA, Brown SC, Potter AC, Metzinger L, Watt DJ, Dickson JG, Tinsley JM, Davies KE: Utrophin-dystrophin-deficient mice as a model for Duchenne muscular dystrophy. Cell 1997, 90:717-727.

39. Baban D, Davies KE: Microarray analysis of $\mathbf{m d x}$ mice expressing high levels of utrophin: therapeutic implications for dystrophin deficiency. Neuromuscul Disord 2008, 18:239-247.

40. Silva C, Moon JC, Elkington AG, John AS, Mohiaddin RH, Pennell DJ: Myocardial late gadolinium enhancement in specific cardiomyopathies by cardiovascular magnetic resonance: a preliminary experience. I Cardiovasc Med (Hagerstown) 2007, 8:1076-1079.

4I. Silva MC, Meira ZM, Gurgel G], da Silva MM, Campos AF, Barbosa MM, Starling Filho GM, Ferreira RA, Zatz M, Rochitte CE: Myocardial delayed enhancement by magnetic resonance imaging in patients with muscular dystrophy. I Am Coll Cardiol 2007, 49:1874-1879.

42. Puchalski MD, Williams RV, Askovich B, Sower CT, Hor KH, Su JT, Pack N, Dibella E, Gottliebson WM: Late gadolinium enhancement: precursor to cardiomyopathy in Duchenne muscular dystrophy? Int J Cardiovasc Imaging 2009, 25:57-63.

43. Gilson WD, Yang Z, French BA, Epstein FH: Measurement of myocardial mechanics in mice before and after infarction using multislice displacement-encoded MRI with 3D motion encoding. Am J Physiol Heart Circ Physiol 2005, 288:HI49I-HI 497.

44. Young AA, Axel L, Dougherty L, Bogen DK, Parenteau CS: Validation of tagging with MR imaging to estimate material deformation. Radiology 1993, 188:101-108. 